Artículo original

\title{
OPTIMIZACIÓN DEL FLUJO VEHICULAR EN LA INTERSECCIÓN VIAL DE LA AV. BOLOGNESI Y LA AV. GUSTAVO PINTO EN LA CIUDAD DE TACNA
}

\author{
OPTIMIZATION OF VEHICLE FLOW IN THE ROAD \\ INTERSECTION OF THE AV. BOLOGNESI AND THE AV. \\ GUSTAVO PINTO IN THE CITY OF TACNA
}

Carla Maricela Urbina Cantuta ${ }^{1}$

Alexa Johana Torres Flores ${ }^{1}$

Rolando Salazar-Calderón Juárez ${ }^{1,2}$

Información del artículo: Recibido: 08/03/2018.

Aceptado: 15/05/2018

${ }^{1}$ Ingeniero Civil+

${ }^{2}$ Docente Facultad de Ingeniería en Universidad Privada de Tacna

INGENIERÍA

Vol. $1, N^{\circ} 1$. 
Julio - Diciembre del 2019.

Ingeniería investiga Vol. 1 (1) Julio - diciembre 2019. 


\section{Resumen}

La presente investigación hace un análisis de las condiciones actuales que se generan debido a la congestión vehicular en la intersección de las dos avenidas principales arteriales en estudio que se dan en horas pico. La finalidad del presente estudio es la de optimizar el comportamiento del flujo vehicular con la presentación de propuestas de solución que mejoren el nivel de servicio actual y que disminuya en la zona de estudio la congestión vehicular existente.

Palabras claves: optimización, flujo vehicular, grado de saturación, nivel de servicio, conteo vehicular, hora pico, simulación, intersección vial, congestión, tráfico.

\section{Abstract}

The present investigation makes an analysis of the current conditions that are generated due to vehicular congestion at the intersection of the two main arterial avenues under study that occur at peak hours. The purpose of the present study is to optimize the behavior of the vehicular flow with the presentation of proposals for solutions that improve the current level of service and reduce the existing vehicular congestion in the study area.

Keywords: optimization, vehicle flow, degree of saturation, service level, vehicle count, rush hour, simulation, road intersection, congestion, traffic.

\section{INTRODUCCIÓN}

El aumento notorio de la demanda vehicular en la última década debido al desarrollo económico del país ha ocasionado problemas y dificultades que tienen las personas al momento de transitar por las calles en las diversas ciudades del ámbito nacional. Este dilema no es indiferente a la ciudad de Tacna, donde los embotellamientos vehiculares son ya una costumbre para los habitantes. En intersecciones sobre todo de avenidas principales, la infraestructura vial es deficiente en lo concerniente al diseño, a la semaforización, así como también a las señales de tránsito, etc. Por estas razones, uno de los propósitos principales de esta investigación es elaborar un estudio vial correcto, construyendo una simulación del tráfico actual para poder hacer un análisis global sobre el nivel de servicio que ofrece la intersección vial de la Av. Bolognesi con Av. Gustavo Pinto. La congestión es un problema considerado como caso crítico, que para mantenerlo controlado y poder garantizar niveles de servicio adecuados, se debe planificar una mejor infraestructura vial, gestión del tráfico y el mejoramiento de hábitos de conducción. Puede esperarse mejores resultados de la intervención progresiva y simultánea en una gran serie de aspectos que integran el sistema de transporte: una adecuada conservación y demarcación de las calles, el mejoramiento de los hábitos de conducción, la coordinación de los tiempos del semáforo, la distribución del transporte público, el mayor énfasis de los volúmenes altos de tráfico producidos por el crecimiento poblacional, entre otros aspectos. 
En la ciudad de Tacna el transporte público está organizado en 33 rutas de transporte, en las que operan 949 vehículos (combis y minibuses), a esto se suma las unidades que realizan el servicio de Taxi generando una gran congestión de las vías principales de la ciudad; sobre todo en las horas pico. El incremento del parque automotor de Tacna en los últimos 7 años ha incrementado la cantidad de semáforos de 29 unidades al año 2007 a 79 unidades al año 2013, que representa más del 250 \%. Arturo Cervantes indicó que en el Perú existe un estilo de conducción muy agresivo y peligroso "que seguramente está costando miles de vidas.

Rodriguez, N. (2015) refiere que es necesario mejorar el nivel de servicio de avenidas, las soluciones deben ser la respuesta al análisis de todos los factores que intervienen. Thomson, I., Bull, A. (2002) afirma que las consecuencias originadas por los mismos operadores se resumen en costos de operación en incremento y mayor tiempo de desplazamiento, viéndose perjudicados, los usuarios del transporte colectivo y los causantes del problema. Calderón, J. (2001) propuso diferentes alternativas convenientes para el método de medición del peso de las variables que intervienen en la ejecución de un proyecto vial urbano las cuales fueron: efecto barrero, compatibilidad de la intersección con deprimidos e intercambios a nivel, seguridad, capacidad, velocidad de operación, uniformidad operacional, costos de operación, costos de capital, disturbios en el tráfico, mantenimiento del tráfico, características estéticas, etc. BLAIR, C., Guevara D. (2012) con la microsimulación con el software SIDRA INTERSECTION 5.1, se realizó un análisis más detallado del comportamiento del flujo vehicular. Ruiz De Somocursio, A. (2008) describe la situación actual del tráfico vehicular en el Perú como deficiente, con sistemas de control de tránsito insuficientes, en la mayoría de las avenidas principales de la capital. Gonzales, D., Rey V. (2016), afirma que la calidad del funcionamiento de las intersecciones viales es determinada por 3 aspectos: la demanda, reflejada en la cantidad de vehículos, la oferta, siendo ésta la capacidad, y los dispositivos de control utilizados en el sitio de análisis. REYNA, P. (2015), determinó niveles de servicio en las avenidas como indicadores de evaluación. Propone el aumento del ciclo verde efectivo en el semáforo en una avenida. Nuñez, Ch., Villanueva C. (2014), lograron resultados favorables, con un nivel de servicio $E$, al aplicar un nuevo diseño de la intersección y bajo la optimización de semáforos utilizando SYNCHRO como software. Según Reyna (2015), el Volumen de tráfico se define como el número de vehículos que circulan por un punto en alguna vía, ya sea por un sentido de vía, un cruce o intersección.

Por esas cuestiones se planteó responder a los siguientes objetivos: Determinar el grado de saturación de la intersección de la Av. Bolognesi con Av. Gustavo Pinto en la ciudad de Tacna; conocer el nivel de servicio existente en la intersección y evaluar el cambio de fases de semaforización y otras propuestas de mejora en la intersección

\section{METODOLOGÍA}

Investigación explicativa y aplicativa mediante el diseño de campo (Vargas, Z. (2009)). La metodología utilizada estuvo basada en el Manual de Capacidad de Carreteras (HCM 2010). La 
información sobre los conteos vehiculares se consiguió mediante el aforo manual desarrollado en el cruce de las avenidas Bolognesi con Gustavo Pinto y durante la fase de recopilación de datos se obtuvo información de trabajo en campo y se solicitó información a la Municipalidad Provincial de Tacna. La información conseguida fue procesada en base a la metodología del Manual HCM. De la misma manera, se usó como instrumento esencial el programa de simulación del tráfico Synchro Traffic 8.0. Para finalizar, se propuso soluciones que estén conforme al estado actual de la intersección con un enfoque crítico y técnico. Se realizó el conteo de tráfico en la intersección durante 7 días. Los días de conteo se realizaron en intervalos de 2 horas por la mañana, 2 horas por la tarde y 2 horas por la noche, agrupados en intervalos de 15 minutos respectivamente. Se seleccionaron las horas pico:

- Sábado 09/12/2017: De 12:30 a 01:30 p.m. con un total de 2184 veh/h.

- Domingo 10/12/2017: De 12:30 a 01:30 p.m. con un total de 1540 veh/h.

- Lunes 11/12/2017: De 07:15 a 08:15 a.m. con un total de 2883 veh/h.

- Martes 12/12/2017: De 07:15 a 08:15 a.m. con un total de $2553 \mathrm{veh} / \mathrm{h}$

- Miércoles13/12/2017: De 07:30 a 08:30 a.m. con un total de 2502 veh/h.

- Jueves 14/12/2017: De 07:30 a 08:30 a.m. con un total de $2494 \mathrm{veh} / \mathrm{h}$

- Viernes 15/12/2017: De 07:30 a 08:30 a.m. con un total de 2342 veh/h.

Asimismo, cada tipo de vehículo se agrupó según el giro codificado que le correspondía (C1, C2, C3, C4, C5, C6, C7, C8, C9, C10, C11, C12) (Fig.1)

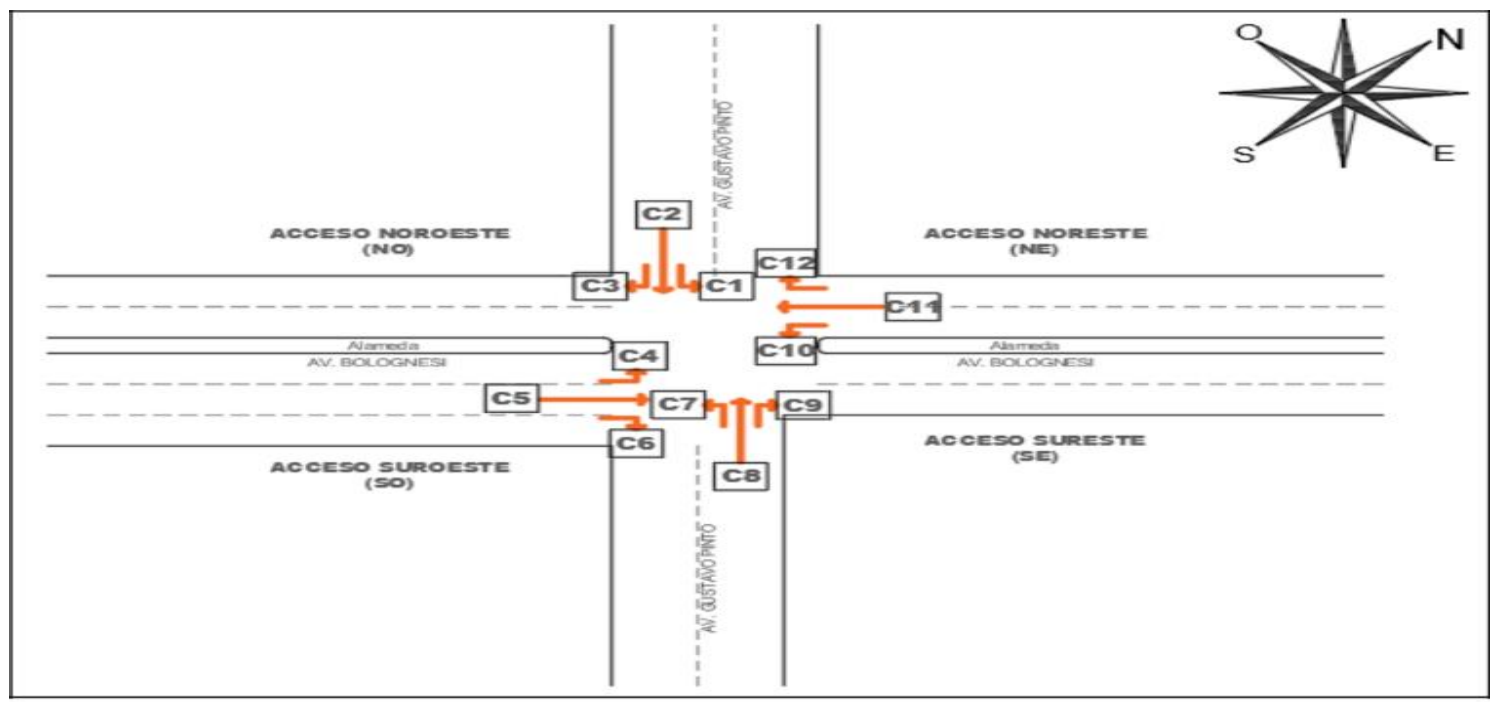

Figura 1: Movimientos identificados en cada acceso

Para efectos de iniciar la simulación de la situación con el programa Synchro fueron necesarios algunos datos adicionales como el porcentaje de vehículos pesados por cada acceso según sus movimientos, los vehículos de transporte público que paran por cada acceso, también el número 
de personas y bicicletas que cruzan la intersección. El número de peatones que transitaron por los diferentes accesos de la intersección fueron contabilizados mediante un aforo manual en campo.

\section{RESULTADOS}

Tabla 1: Demora total y alternativas de solución

\begin{tabular}{cccc}
\hline Alternativa de solución & Grado de saturación & Demora total & Nivel de servicio \\
\hline $\mathrm{N}^{\circ} 01$ & 1.28 & 163.6 segundos & $\mathrm{F}$ \\
$\mathrm{N}^{\circ} 02$ & 1.69 & 151.8 segundos & $\mathrm{F}$ \\
$\mathrm{N}^{\circ} 03$ & 1.41 & 137.7 segundos & $\mathrm{F}$ \\
$\mathrm{N}^{\circ} 04$ & 0.99 & 45.5 segundos & $\mathrm{D}$ \\
$\mathrm{N}^{\circ} 05$ & 0.99 & 49.4 segundos & $\mathrm{D}$ \\
$\mathrm{N}^{\circ} 06$ & 0.72 & 10.5 segundos & $\mathrm{B}$ \\
$\mathrm{N}^{\circ} 07$ & 0.51 & 8.3 segundos & $\mathrm{A}$ \\
\hline
\end{tabular}

El resultado obtenido muestra el grado de saturación crítico o la relación volumen a capacidad máxima, obtenida en la simulación actual del tráfico en la intersección, al ser mayor que 1, nos indica un exceso de demanda de vehículos sobre la capacidad que estas vías tienen. Éste resultado es importante ya que el grado de saturación crítico hace un análisis global a la intersección, referido al ciclo semafórico y a su geometría, tomando en cuenta únicamente los accesos críticos, los cuales tienen la relación de flujo más alta en cada fase. Esta relación nos sirve para poder tener en cuenta que se debe hacer un análisis de la capacidad de las vías de carriles simples o agrupados con respecto a sus movimientos críticos ya que no están cumpliendo con relación al tráfico vehicular actual, produciendo congestión vehicular.

\section{DISCUSIÓN}

Se confirma, que el grado de saturación es altamente saturado debido a la gran cantidad de vehículos que se presenta en la intersección de la Av. Bolognesi con Av. Gustavo Pinto en la ciudad de Tacna. El resultado obtenido muestra el nivel de servicio de la intersección, obtenido mediante el análisis del tráfico vehicular real y una simulación hecha con el software Synchro Traffic 8.0, al ser F, nos indica un fuerte nivel de congestión vehicular debido a altas demoras, ya que es superior a 80 segundos y un grado de saturación mayor a 1 ya que, en los accesos en la intersección, los flujos que llegan superan la capacidad. Éste resultado nos permite tener conocimiento del nivel de servicio actual, que está basada en la demora media de vehículos parados por la señal del semáforo y la relación volumen a capacidad. La desventaja que presenta actualmente la intersección es que el ciclo semafórico tiene un periodo muy largo, por ejemplo, cuando se pone en tiempo verde en uno de los accesos, el resto de las calles todas están en tiempo rojo y tienen que esperar a que pasen los otros tres accesos para poder pasar. La ventaja es que los vehículos mixtos pueden voltear en 3 sentidos, a la izquierda, a la derecha o ir de 
frente. Asimismo, la otra ventaja que se presenta es que estando en tiempo rojo se puede poner un semáforo direccional con una flecha que indica que se puede girar a la derecha estando en tiempo rojo. Lamentablemente esta distribución a 4 fases no permite la fluidez del tráfico vehicular por lo cual debería ser modificado.

Las propuestas de mejora es la construcción del paso a desnivel en el acceso Suroeste y Noreste, la modificación de la sección vial con el aumento de carriles en el acceso Noroeste y Sureste. La segunda propuesta es el cambio de distribución de 4 fases a 2 fases de los semáforos ya que es cuestión de programación técnica de los semáforos. La tercera propuesta es el cambio de distribución de 4 fases a 2 fases de los semáforos y la modificación de la sección vial con el aumento de carriles en la Av. Gustavo Pinto tanto en el acceso Sureste y Noroeste, también en la Av. Bolognesi en el acceso Suroeste eliminar el estacionamiento para ampliar la longitud del tercer carril. Se recomienda a la Sub Gerencia de Transporte Público y Tránsito, la implementación de semáforos inteligentes y/o contadores podrían ser también una posible manera de ordenar el tráfico y dar prioridad a los accesos con más demanda vehicular.

\section{REFERENCIAS BIBLIOGRÁFICAS}

Alcalá, M. (2016). Micro-simulación del tráfico de la intersección de las Avenidas Bolívar, Córdova y calle Andalucía empleando el software Vissim 6.

Alvarez, J. (2017). Micro-simulación intermodal en la ciudad del Cusco empleando los softwares Vissim 8 y Viswalk 8.

Angaspilco, Ch. (2014). Nivel de serviciabilidad en las Avenidas; Atahualpa, Juan XXII, Independencia, de los Héroes y San Martín de la ciudad de Cajamarca.

Asociacion BCEOM - OIST. (2001). Manual de Tránsito.

Ballon, S. (2016). Estudio, Simulación y Optimización del Flujo De Tráfico de la Av. Jorge Chávez desde la Calle Víctor Lira hasta la Calle Paucarpata.

Bayona, B., Marquez, T. (2015). La congestión vehicular en la ciudad de Piura.

Cal Y Mayor, R., Cardenas, J. (2007). Ingeniería de Transito - Fundamentos y aplicaciones. 8va Edición. México D.F., México. Alfaomega Grupo Editor S.S. de C.V.

Cavagnaro, L. (2000). Tacna: Desarrollo Urbano y Arquitectónico (1536-1880).

Diaz, L. (2009). Análisis vial de dos intersecciones sin semáforo en zona aledaña a nuevo Terrapuerto de Piura.

Esquivel, W. (2011). Elementos de diseño y planeamiento de intersecciones urbanas.

Flores, Y. (2016). Micro-simulación de la intersección de la Avenida Universitaria con la Avenida Bolívar utilizando los softwares Vissim y Viswalk 8.

Fuentes, L., Sueros, W. (2013). Diseño geométrico y diseño estructural del intercambio vial en la intersección de la Av. Alfonso Ugarte y la Av. Miguel de Forja en el cercado de Arequipa. 
Gonzales, D., Rey, V. (2016). Propuesta de mejora de los niveles de servicio para mitigar la congestión vehicular en las intersecciones de la Av. Rafael Escardo comprendida entre las avenidas Costanera, La Paz y La Libertad, Lima

- San Miguel.

González, J., Rodriguez, G., Martinez, J. (2000) ¿Cómo mejorar el flujo vehicular por medio de la simulación?

MTC. (2016). Manual de Dispositivos de Control de Tránsito Automotor para calles y carreteras.

Nuñez, Ch., Villanueva, C. (2014). Solución Vial de la Av. Primavera comprendida entre las Avenidas La Encalada y José Nicolás Rodrigo, Lima-Lima- Surco.

Padilla, C., Ulloa, A. (2016). Diseño y planeamiento de una intersección vial urbana no semaforizada simulando interacción con el área de influencia.

Pinto, Ch. (2016). Análisis y planteamiento de soluciones en el ovalo "Los Incas" - intersección de la Avenida Dolores con la Avenida Los Incas en la Provincia de Arequipa.

Reyna, P. (2015). Propuesta de mejora de niveles de servicio en dos intersecciones.

Rodríguez, D. (2015). Revisión del Hcm 2010 y 2000 Intersecciones Semaforizadas, Ingenium, vol. $16,{ }^{\circ} 32$, pp. $19-31$.

Rodríguez, N. (2015). Evaluación del congestionamiento vehicular en intersecciones viales.

Ruiz, A. (2008). Control de tráfico vehicular automatizado utilizando lógica difusa.

Ruiz, S. (2012). Evaluación del flujo vehicular en la intersección de la carrera 70 con la calle 9 por la construcción de una vía subterránea en el aeropuerto Enrique Olaya Herrera.

Soto, A. (2017). Rediseño vial de la Avenida Mariano Cornejo entre la calle Santa Bárbara y el Jirón Saturno.

Suarez, L. (2007). Análisis y evaluación operacional de intersecciones urbanas mediante microsimulación.

Thomson, I., Bull, A. (2002). La congestión del tránsito urbano: causas y consecuencias económicas y sociales. Revista de la Cepal 76.

Trafficware. (2011). Manual Synchro Studio 8.

Transportation Research Bord. (2010). Manual de Capacidad de Carreteras.

Vchi.SA. (2005). Manual de Diseño Geométrico de Vías Urbanas.

Velasco, J. (2017). Los estudios de impacto vial y el tráfico generado en la ciudad de Lima.

Vera, F. (2012). Aplicabilidad de las metodologías del HCM 2000 y Synchro 7.0 para analizar intersecciones semaforizadas en Lima. 